\title{
Faster than Hermitian Time Evolution ${ }^{\star}$
}

Carl M. BENDER

Physics Department, Washington University, St. Louis, MO 63130, USA

E-mail: cmb@wustl.edu

URL: http://www.physics.wustl.edu/ cmb/

Received October 22, 2007, in final form December 22, 2007; Published online December 26, 2007

Original article is available at http://www.emis.de/journals/SIGMA/2007/126/

\begin{abstract}
For any pair of quantum states, an initial state $|I\rangle$ and a final quantum state $|F\rangle$, in a Hilbert space, there are many Hamiltonians $H$ under which $|I\rangle$ evolves into $|F\rangle$. Let us impose the constraint that the difference between the largest and smallest eigenvalues of $H$, $E_{\max }$ and $E_{\min }$, is held fixed. We can then determine the Hamiltonian $H$ that satisfies this constraint and achieves the transformation from the initial state to the final state in the least possible time $\tau$. For Hermitian Hamiltonians, $\tau$ has a nonzero lower bound. However, among non-Hermitian $\mathcal{P} \mathcal{T}$-symmetric Hamiltonians satisfying the same energy constraint, $\tau$ can be made arbitrarily small without violating the time-energy uncertainty principle. The minimum value of $\tau$ can be made arbitrarily small because for $\mathcal{P} \mathcal{T}$-symmetric Hamiltonians the path from the vector $|I\rangle$ to the vector $|F\rangle$, as measured using the Hilbert-space metric appropriate for this theory, can be made arbitrarily short. The mechanism described here is similar to that in general relativity in which the distance between two space-time points can be made small if they are connected by a wormhole. This result may have applications in quantum computing.
\end{abstract}

Key words: brachistochrone; PT quantum mechanics; parity; time reversal; time evolution; unitarity

2000 Mathematics Subject Classification: 81Q10; 81S99

\section{Classical brachistochrone problem}

Three hundred years ago the solution to a famous problem in classical mechanics known as the brachistochrone was found almost simultaneously by a number of distinguished mathematicians including Newton, Bernoulli, Leibniz, and L'Hôpital. (The term brachistochrone is derived from Greek and means shortest time.) The problem is stated as follows: A bead slides down a frictionless wire from one given point to another in a homogeneous gravitational field. What is the shape of the wire connecting the two points that minimizes the time of descent of the bead? The solution found by these mathematicians is that the wire must be in the shape of a cycloid.

Of course, it is implicitly assumed in the derivation of the brachistochrone that the path of shortest time of descent is real. It is interesting that if one allows for the possibility of complex paths of motion, one can achieve an even shorter time of flight.

To illustrate how shorter times can be achieved by means of complex paths, let us consider the simple classical harmonic oscillator, whose Hamiltonian is given by

$$
H=p^{2}+x^{2} .
$$

If we have a particle of energy $E=1$, then the classical turning points of the motion of the particle are located at $x= \pm 1$. The particle undergoes simple harmonic motion in which it

${ }^{\star}$ This paper is a contribution to the Proceedings of the Seventh International Conference "Symmetry in Nonlinear Mathematical Physics" (June 24-30, 2007, Kyiv, Ukraine). The full collection is available at http://www.emis.de/journals/SIGMA/symmetry2007.html 
oscillates sinusoidally between these two turning points. This periodic motion is indicated in Fig. 1 by a solid line connecting the turning points. However, in addition to this oscillatory motion on the real- $x$ axis, there are an infinite number of other trajectories that a particle of energy $E$ can have [1]. These classical trajectories, which are also shown in Fig. 1, are all ellipses whose foci are located at precisely the positions of the turning points. All of the classical orbits are periodic and all orbits have the same period $T=2 \pi$. Thus, a classical particle travels faster along more and more distant ellipses.

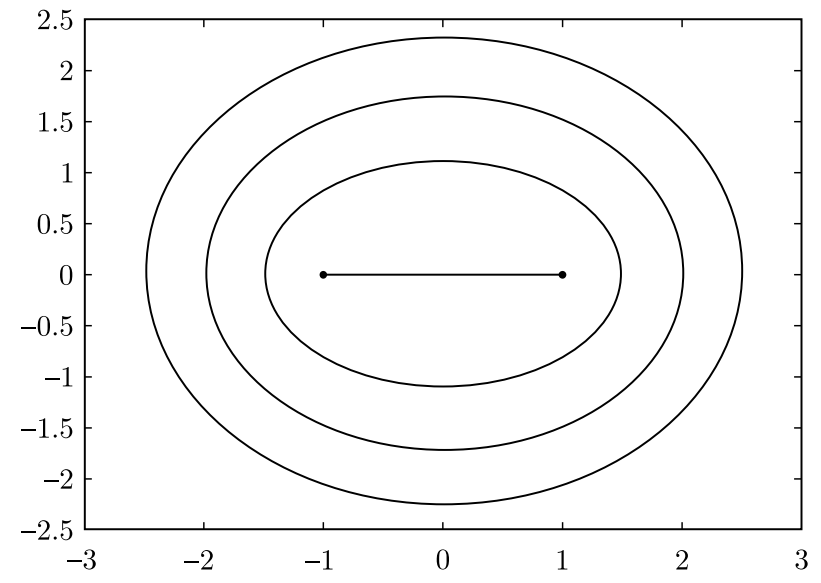

Figure 1. Classical trajectories in the complex- $x$ plane for the harmonic oscillator whose Hamiltonian is $H=p^{2}+x^{2}$. These trajectories represent the possible paths of a particle whose energy is $E=1$. The trajectories are nested ellipses with foci located at the turning points at $x= \pm 1$. The real line segment (degenerate ellipse) connecting the turning points is the usual periodic classical solution to the harmonic oscillator. All closed paths have the same period $2 \pi$.

Now suppose that a classical particle of energy $E=1$ is traveling along the real- $x$ axis from some point $x=-a$ to $x=a$, where $a>1$. If the potential $V$ is everywhere zero along its path, then it will travel at a constant velocity. However, if the particle suddenly finds itself in the parabolic potential $V(x)=x^{2}$ just as it reaches the turning point at $x=-1$ and it suddenly escapes the influence of this potential at $x=1$, then the time of flight from $x=-a$ to $x=a$ will be changed because the particle is not traveling at constant velocity between the turning points. Now imagine that the potential $V(x)=x^{2}$ is suddenly turned on before the particle reaches the turning point at $x=-1$. In this case, the particle will follow one of the elliptical paths in the complex plane around to the positive real axis. Just as the particle reaches the positive real axis the potential is turned off, so the particle proceeds onward along the real axis until it reaches $x=a$. This trip will take less time because the particle travels faster along the ellipse in the complex plane.

We have arrived at the surprising conclusion that if the classical particle enters the parabolic potential $V(x)=x^{2}$ immediately after it begins its voyage up the real axis, its time of flight will be exactly half a period, or $\pi$. Indeed, by traveling in the complex plane, a particle of energy $E=1$ can go from the point $x=-a$ to the point $x=a$ in time $\pi$, no matter how large $a$ is. Evidently, if a particle is allowed to follow complex classical trajectories, then it is possible to make a drastic reduction in its time of flight between two given real points.

\section{Quantum brachistochrone problem}

The purpose of this paper is to show that by using complex non-Hermitian Hamiltonians, we can obtain a faster time of flight than is possible with Hermitian Hamiltonians. The quantum 
brachistochrone problem is defined as follows: If we are given an initial quantum state $|I\rangle$ and a final quantum state $|F\rangle$, then there exist many Hamiltonians $H$ under which $|I\rangle$ evolves into $|F\rangle$. The quantum brachistochrone problem is to find the particular Hamiltonian $H$ that achieves this transformation in the least time $\tau$, subject to the constraint that the difference between the largest and smallest eigenvalues of $H$ is held fixed. For Hermitian Hamiltonians, $\tau$ has a nonzero lower bound. However, we will see that for non-Hermitian $\mathcal{P} \mathcal{T}$-symmetric Hamiltonians satisfying the same energy constraint, $\tau$ can be made arbitrarily small.

One might think that this result could violate the time-energy uncertainty principle. However, we will see that this is not the case because for non-Hermitian Hamiltonians the path from $|I\rangle$ to $|F\rangle$ can be made arbitrarily short. The mechanism is similar to that in general relativity, where the distance between two space-time points can be made small if they are connected by a wormhole.

\section{$3 \quad$ Review of $\mathcal{P} \mathcal{T}$ quantum mechanics}

Based on the traditional training that one receives in a quantum mechanics course, one would expect a theory defined by a non-Hermitian Hamiltonian to be unphysical because the energy levels would most likely be complex and the time evolution would most likely be nonunitary (not probability-conserving). However, theories defined by a special class of non-Hermitian Hamiltonians called $\mathcal{P} \mathcal{T}$-symmetric Hamiltonians can have positive real energy levels and can exhibit unitary time evolution. Such theories are acceptable quantum theories. In principle, these theories can be distinguished experimentally from those defined by Hermitian Hamiltonians because non-Hermitian time evolution can proceed arbitrarily rapidly.

We use the following notation in this paper: By the term Hermitian, we mean Dirac Hermitian, where the Dirac Hermitian adjoint symbol ${ }^{\dagger}$ represents combined matrix transposition and complex conjugation. The parity operator $\mathcal{P}$ performs spatial reflection $x \rightarrow-x$ and the antilinear time-reversal operator $\mathcal{T}$ performs combined time reversal $t \rightarrow-t$ and complex conjugation.

The first $\mathcal{P} \mathcal{T}$-symmetric quantum-mechanical Hamiltonians were introduced in 1998 [2]; and since then there have been many follow-up papers by a wide range of authors. There have also been three recent review articles [3, 4, 5]. In [2] it was discovered that even if a Hamiltonian is not Hermitian, its energy levels can be all real and positive so long as the eigenfunctions are symmetric under $\mathcal{P} \mathcal{T}$ reflection.

These new kinds of Hamiltonians are obtained by deforming ordinary Hermitian Hamiltonians into the complex domain. The original class of $\mathcal{P} \mathcal{T}$-symmetric Hamiltonians that was proposed in [2] has the form

$$
H=p^{2}+x^{2}(i x)^{\epsilon} \quad(\epsilon>0),
$$

where $\epsilon$ is a real deformation parameter. Two particularly interesting special cases are obtained by setting $\epsilon=1$ to obtain $H=p^{2}+i x^{3}$ and by setting $\epsilon=2$ to obtain $H=p^{2}-x^{4}$. Surprisingly, these Hamiltonians have real, positive, discrete energy levels even though the potential for $\epsilon=1$ is imaginary and the potential for $\epsilon=2$ is upside-down. The first proof of spectral reality and positivity for $H$ in (1) was given by Dorey et al. in [6].

The philosophical background of $\mathcal{P} \mathcal{T}$ quantum mechanics is simply this: The axiom of quantum mechanics that requires the Hamiltonian $H$ to be Dirac Hermitian is distinct from all of the other axioms because it is mathematical in character rather than physical. The other axioms are stated in physical terms; these other axioms require locality, causality, stability and uniqueness of the vacuum state, conservation of probability, Lorentz invariance, and so on. The condition of Dirac Hermiticity $H=H^{\dagger}$ is mathematical, but the condition of $\mathcal{P} \mathcal{T}$ symmetry $H=H^{\mathcal{P} \mathcal{T}}$ (space-time reflection symmetry) is physical because $\mathcal{P}$ and $\mathcal{T}$ are elements of the Lorentz group. 
The spectrum $H$ in (1) is real, which poses the question of whether this Hamiltonian specifies a quantum-mechanical theory. That is, is the theory specified by $H$ associated with a Hilbert space endowed with a positive inner product and does $H$ specify unitary (norm-preserving) time evolution? The answer to these questions is yes. Positivity of the inner product and unitary time evolution was established in [7] for quantum-mechanical systems having an unbroken $\mathcal{P} \mathcal{T}$ symmetry and in [8] for quantum field theory.

To demonstrate that the theory specified by the $H$ in (1) is a quantum-mechanical theory, we construct a linear operator $\mathcal{C}$ that satisfies the three simultaneous algebraic equations [7]: $\mathcal{C}^{2}=1,[\mathcal{C}, \mathcal{P} \mathcal{T}]=0$, and $[\mathcal{C}, H]=0$. Using $\mathcal{C}$, which in quantum field theory is a Lorentz scalar [9], we can then construct the appropriate inner product for a $\mathcal{P} \mathcal{T}$-symmetric Hamiltonian: $\langle a \mid b\rangle \equiv a^{\mathcal{C P} \mathcal{T}} \cdot b$. This inner product, which uses the $\mathcal{C P} \mathcal{T}$ adjoint, has a strictly positive norm: $\langle a \mid a\rangle>0$. Because $H$ commutes with both $\mathcal{P} \mathcal{T}$ and $\mathcal{C}, H$ is self-adjoint with respect to $\mathcal{C P} \mathcal{T}$ conjugation. Also, the time-evolution operator $e^{-i H t}$ is unitary with respect to $\mathcal{C} \mathcal{P} \mathcal{T}$ conjugation. Note that the Hilbert space and the $\mathcal{C P} \mathcal{T}$ inner product is dynamically determined by the Hamiltonian itself.

We have explained why a $\mathcal{P} \mathcal{T}$-symmetric Hamiltonian gives rise to a unitary theory, but in doing so we raise the question of whether $\mathcal{P} \mathcal{T}$-symmetric Hamiltonians are useful. The answer to this question is simply that $\mathcal{P} \mathcal{T}$-symmetric Hamiltonians have already been useful in many areas of physics. For example, in $1959 \mathrm{Wu}$ showed that the ground state of a Bose system of hard spheres is described by a non-Hermitian Hamiltonian [10]. Wu found that the ground-state energy of this system is real and he conjectured that all of the energy levels were real. Hollowood showed that the non-Hermitian Hamiltonian for a complex Toda lattice has real energy levels [11]. Cubic non-Hermitian Hamiltonians of the form $H=p^{2}+i x^{3}$ (and also cubic quantum field theories having an imaginary self-coupling term) arise in studies of the Lee-Yang edge singularity [12] and in various Reggeon field-theory models [13]. In all of these cases a non-Hermitian Hamiltonian having a real spectrum appeared mysterious at the time, but now the explanation is simple: In every case the non-Hermitian Hamiltonian is $\mathcal{P} \mathcal{T}$ symmetric. Hamiltonians having $\mathcal{P} \mathcal{T}$ symmetry have also been used to describe magnetohydrodynamic systems [14] and to study nondissipative time-dependent systems interacting with electromagnetic fields [15].

An important application of $\mathcal{P} \mathcal{T}$ quantum mechanics is in the revitalization of theories that have been abandoned because they appear to have ghosts. Ghosts are states having negative norm. We have explained above that in order to construct the quantum-mechanical theory defined by a $\mathcal{P} \mathcal{T}$-symmetric Hamiltonian, we must construct the appropriate adjoint from the $\mathcal{C}$ operator. Having constructed the $\mathcal{C P} \mathcal{T}$ adjoint, one may find that the so-called ghost state is actually not a ghost at all because when its norm is calculated using the correct definition of the adjoint, the norm turns out to be positive. This is precisely what happens in the case of the Lee model.

The Lee model was proposed in 1954 as a quantum field theory in which mass, wave-function, and charge renormalization could be performed exactly and in closed form [16]. However, in 1955 Källén and Pauli showed that when the renormalized coupling constant is larger than a critical value, the Hamiltonian becomes non-Hermitian (in the Dirac sense) and a ghost state appears [17]. The appearance of the ghost was assumed to be a fundamental defect of the Lee model. However, the non-Hermitian Lee-model Hamiltonian is $\mathcal{P} \mathcal{T}$ symmetric and when the norms of the states of this model are determined using the $\mathcal{C}$ operator, which can be calculated in closed form, the ghost state is seen to be an ordinary physical state having positive norm [18]. Thus, the following words by Barton [19] are not true: "A non-Hermitian Hamiltonian is unacceptable partly because it may lead to complex energy eigenvalues, but chiefly because it implies a non-unitary $\mathrm{S}$ matrix, which fails to conserve probability and makes a hash of the physical interpretation." 
Another example of a quantum model that was thought to have ghost states, but in fact does not, is the Pais-Uhlenbeck oscillator model [20]. This model has a fourth-order field equation, and for the past several decades it was thought (incorrectly) that all such higher-order field equations lead inevitably to ghosts. Indeed, it is explained in [20] when the Pais-Uhlenbeck model is quantized using the methods of $\mathcal{P} \mathcal{T}$ quantum mechanics, it does not have any ghost states at all.

There are many potential applications for $\mathcal{P} \mathcal{T}$ quantum mechanics in areas such as particle physics, cosmology, gravitation, quantum field theory, and solid-state physics. These applications are discussed in detail in the recent review article [4].

Having established the validity and potential usefulness of $\mathcal{P} \mathcal{T}$ quantum mechanics, one may ask why $\mathcal{P} \mathcal{T}$ quantum mechanics works. The reason is that $\mathcal{C P}$ is a positive operator, and thus it can be written as the exponential of another operator $\mathcal{Q}: \mathcal{C P}=e^{\mathcal{Q}}$. The square root of $e^{\mathcal{Q}}$ can then be used to construct a new Hamiltonian $\tilde{H}$ via a similarity transformation on the $\mathcal{P} \mathcal{T}$-symmetric Hamiltonian $H: \tilde{H} \equiv e^{-\mathcal{Q} / 2} H e^{\mathcal{Q} / 2}$. The new Hamiltonian $\tilde{H}$ has the same energy eigenvalues as the original Hamiltonian $H$ because a similarity transformation is isospectral. Moreover, $\tilde{H}$ is Dirac Hermitian [21]. $\mathcal{P} \mathcal{T}$ quantum mechanics works because there is an equivalence between a non-Hermitian $\mathcal{P} \mathcal{T}$-symmetric Hamiltonian and a conventional Dirac Hermitian Hamiltonian.

There are a number of elementary examples of this equivalence, but a nontrivial illustration is provided by the Hamiltonian $H$ in (1) at $\epsilon=2$, which is not Hermitian because boundary conditions that violate the $L^{2}$ norm must be imposed in Stokes wedges in the complex plane in order to obtain a real, positive, discrete spectrum. The exact equivalent Hermitian Hamiltonian is $\tilde{H}=p^{2}+4 x^{4}-2 \hbar x$, where $\hbar$ is Planck's constant [22]. The term proportional to $\hbar$ vanishes in the classical limit and is thus an example of a quantum anomaly.

We have established that $\mathcal{P} \mathcal{T}$ symmetry is equivalent by means of a similarity transformation to conventional Dirac Hermiticity. Therefore, one may wonder whether $\mathcal{P} \mathcal{T}$ quantum mechanics is actually fundamentally different from ordinary quantum mechanics? The answer is yes, and this paper argues that, at least in principle, there is an experimentally observable difference between $\mathcal{P} \mathcal{T}$-symmetric and ordinary Dirac Hermitian Hamiltonians. The quantum brachistochrone provides a setting for examining this difference and provides a way to discriminate between the class of $\mathcal{P} \mathcal{T}$-symmetric Hamiltonians and the class of Dirac Hermitian Hamiltonians.

\section{Solving the Hermitian quantum brachistochrone problem}

To find the Hermitian Hamiltonian $H$ that solves the quantum brachistochrone problem we must examine all possible Hamiltonians under which a state $\left|\psi_{I}\right\rangle$ in Hilbert space evolves into another state $\left|\psi_{F}\right\rangle$ in time $t$ :

$$
\left|\psi_{I}\right\rangle \rightarrow\left|\psi_{F}\right\rangle=e^{-i H t / \hbar}\left|\psi_{I}\right\rangle
$$

The problem is to find the minimum time $t=\tau$ required for this transformation, subject to the constraint that the difference $\omega$ between the largest and smallest eigenvalues of $H$,

$$
\omega=E_{\max }-E_{\min },
$$

is held fixed. The quantum brachistochrone is the Hamiltonian that performs this time evolution in the least possible time. In [23] it is shown that for Hermitian Hamiltonians $\tau \neq 0$. However, we show in this paper that one can find a Hamiltonian in the space of $\mathcal{P} \mathcal{T}$-symmetric Hamiltonians that satisfies the same energy constraint and can perform the time evolution in no time at all! 
Here, we study the simplest case of Hamiltonians having only two energy levels. We restrict the discussion to this case because it is shown in [24] that one need only work in the twodimensional subspace of the full Hilbert space that is spanned by the initial state vector $\left|\psi_{I}\right\rangle$ and the final state vector $\left|\psi_{F}\right\rangle$. We consider the case of Hermitian Hamiltonians and choose a basis so that

$$
\left|\psi_{I}\right\rangle=\left(\begin{array}{c}
1 \\
0
\end{array}\right) \quad \text { and } \quad\left|\psi_{F}\right\rangle=\left(\begin{array}{c}
a \\
b
\end{array}\right)
$$

where the condition that $\left|\psi_{F}\right\rangle$ be normalized is $|a|^{2}+|b|^{2}=1$. The most general $2 \times 2$ Hermitian Hamiltonian is

$$
H=\left(\begin{array}{cc}
s & r e^{-i \theta} \\
r e^{i \theta} & u
\end{array}\right) \quad(r, s, u, \theta \text { real }) .
$$

For this Hamiltonian the eigenvalue constraint takes the form

$$
\omega^{2}=(s-u)^{2}+4 r^{2}
$$

To find the optimal Hamiltonian satisfying this constraint, we express $H$ in terms of the Pauli matrices:

$$
H=\frac{1}{2}(s+u) \mathbf{1}+\frac{1}{2} \omega \sigma \cdot \mathbf{n},
$$

where

$$
\mathbf{n}=\frac{1}{\omega}(2 r \cos \theta, 2 r \sin \theta, s-u)
$$

is a unit vector and

$$
\sigma_{1}=\left(\begin{array}{cc}
0 & 1 \\
1 & 0
\end{array}\right), \quad \sigma_{2}=\left(\begin{array}{cc}
0 & -i \\
i & 0
\end{array}\right), \quad \sigma_{3}=\left(\begin{array}{cc}
1 & 0 \\
0 & -1
\end{array}\right) .
$$

We use the identity

$$
\exp (i \phi \sigma \cdot \mathbf{n})=\cos \phi \mathbf{1}+i \sin \phi \sigma \cdot \mathbf{n}
$$

to write $\left|\psi_{F}\right\rangle=e^{-i H \tau / \hbar}\left|\psi_{I}\right\rangle$ as

$$
\left(\begin{array}{l}
a \\
b
\end{array}\right)=e^{-\frac{1}{2} i(s+u) t / \hbar}\left(\begin{array}{c}
\cos \frac{\omega t}{2 \hbar}-i \frac{s-u}{\omega} \sin \frac{\omega t}{2 \hbar} \\
-i \frac{2 r}{\omega} e^{i \theta} \sin \frac{\omega t}{2 \hbar}
\end{array}\right) .
$$

The second component of this equation then gives $|b|=\frac{2 r}{\omega} \sin \frac{\omega t}{2 \hbar}$, which allows us to find the required time of evolution:

$$
t=\frac{2 \hbar}{\omega} \arcsin \frac{\omega|b|}{2 r}
$$

We must now minimize the time $t$ over all $r>0$ while maintaining the constraint in (2). This constraint tells us that the maximum value of $r$ is $\frac{1}{2} \omega$. At this maximum we have $s=u$. The minimum evolution time $\tau$ is thus given by

$$
\tau \omega=2 \hbar \arcsin |b|
$$


Note that if $a=0$ and $b=1$, we have $\tau=\pi \hbar / \omega$ for the smallest time required to transform

$\left(\begin{array}{l}1 \\ 0\end{array}\right)$ to the orthogonal state $\left(\begin{array}{l}0 \\ 1\end{array}\right)$. The time $\tau$ required to transform a vector into an orthogonal vector is called the passage time.

The form of the result in (3) resembles the uncertainty principle, but (3) is merely the statement that rate $\times$ time $=$ distance. The constraint in (2) is equivalent to a bound on the standard deviation $\Delta H$, where $(\Delta H)^{2}=\left\langle\psi\left|H^{2}\right| \psi\right\rangle-\langle\psi|H| \psi\rangle^{2}$ in the normalized state $|\psi\rangle$. The maximum of $\Delta H$ is $\omega / 2$. The speed of evolution of a quantum state is given by $\Delta H$. The distance between the initial state $\left|\psi_{I}\right\rangle$ and the final state $\left|\psi_{F}\right\rangle$ is $2 \arccos \left(\left|\left\langle\psi_{F} \mid \psi_{I}\right\rangle\right|\right)$. Thus, the time $\tau$ to evolve from $\left|\psi_{I}\right\rangle$ to $\left|\psi_{F}\right\rangle$ is bounded below because the speed is bounded above with the distance held fixed.

\section{Solving the non-Hermitian quantum brachistochrone problem}

For a $\mathcal{P} \mathcal{T}$-symmetric Hamiltonian, $\tau$ can be arbitrarily small. This is because a $\mathcal{P} \mathcal{T}$-symmetric Hamiltonian whose eigenvalues are all real is equivalent to a Hermitian Hamiltonian via $\tilde{H}=$ $e^{-\mathcal{Q} / 2} \mathrm{He}^{\mathcal{Q} / 2}$. The states in a $\mathcal{P} \mathcal{T}$-symmetric theory are mapped by $e^{-\mathcal{Q} / 2}$ to the corresponding states in the Dirac Hermitian theory. But, the overlap distance between two states does not remain constant under a similarity transformation. We can exploit this property of the similarity transformation to overcome the Hermitian lower limit on the time $\tau$. The detailed calculation is explained in [25], and this calculation has already led to much research activity and lively debate [26].

We consider the general class of $\mathcal{P} \mathcal{T}$-symmetric $2 \times 2$ Hamiltonians having the form

$$
H=\left(\begin{array}{cc}
r e^{i \theta} & s \\
s & r e^{-i \theta}
\end{array}\right) \quad(r, s, \theta \text { real })
$$

where $\mathcal{T}$ is complex conjugation and $\mathcal{P}=\left(\begin{array}{ll}0 & 1 \\ 1 & 0\end{array}\right)$. The eigenvalues

$$
E_{ \pm}=r \cos \theta \pm \sqrt{s^{2}-r^{2} \sin ^{2} \theta}
$$

are real if $s^{2}>r^{2} \sin ^{2} \theta$. This inequality defines the region of unbroken $\mathcal{P} \mathcal{T}$ symmetry. The unnormalized eigenstates of $H$ are

$$
\left|E_{+}\right\rangle=\left(\begin{array}{c}
e^{i \alpha / 2} \\
e^{-i \alpha / 2}
\end{array}\right), \quad\left|E_{-}\right\rangle=\left(\begin{array}{c}
i e^{-i \alpha / 2} \\
-i e^{i \alpha / 2}
\end{array}\right)
$$

where $\alpha$ (real) is given by $\sin \alpha=(r / s) \sin \theta$. The $\mathcal{C}$ operator for $H$ in (4) is

$$
\mathcal{C}=\frac{1}{\cos \alpha}\left(\begin{array}{cc}
i \sin \alpha & 1 \\
1 & -i \sin \alpha
\end{array}\right) \text {. }
$$

It is easy to verify that the $\mathcal{C P} \mathcal{T}$ norms of both eigenstates have the value $\sqrt{2 \cos \alpha}$.

To calculate $\tau$ we express the $H$ in (4) as

$$
H=(r \cos \theta) \mathbf{1}+\frac{1}{2} \omega \sigma \cdot \mathbf{n},
$$

where

$$
\mathbf{n}=\frac{2}{\omega}(s, 0, i r \sin \theta)
$$

is a unit vector. The squared difference between energy eigenvalues is

$$
\omega^{2}=4 s^{2}-4 r^{2} \sin ^{2} \theta .
$$

The positivity of $\omega^{2}$ is ensured by the condition of unbroken $\mathcal{P} \mathcal{T}$ symmetry. 
To determine $\tau$ we write down the $\mathcal{P} \mathcal{T}$-symmetric time-evolution equation:

$$
e^{-i H t / \hbar}\left(\begin{array}{l}
1 \\
0
\end{array}\right)=\frac{e^{-i t r \cos \theta / \hbar}}{\cos \alpha}\left(\begin{array}{c}
\cos \left(\frac{\omega t}{2 \hbar}-\alpha\right) \\
-i \sin \left(\frac{\omega t}{2 \hbar}\right)
\end{array}\right) .
$$

Consider the pair of vectors used in the Hermitian case: $\left|\psi_{I}\right\rangle=\left(\begin{array}{l}1 \\ 0\end{array}\right)$ and $\left|\psi_{F}\right\rangle=\left(\begin{array}{l}0 \\ 1\end{array}\right)$. (Note that these two vectors are not orthogonal with respect to the $\mathcal{C P} \mathcal{T}$ inner product.) Observe that the evolution time needed to reach $\left|\psi_{F}\right\rangle$ from $\left|\psi_{I}\right\rangle$ is $t=(2 \alpha-\pi) \hbar / \omega$. Optimizing this result over allowable values for $\alpha$ as $\alpha$ approaches $\frac{1}{2} \pi$, the optimal time $\tau$ tends to zero!

\section{Discussion}

Equations (2) and (5) reveal the difference between Hermitian and $\mathcal{P} \mathcal{T}$-symmetric Hamiltonians. Equation (2) for the Hermitian matrix Hamiltonian has a sum of squares while (5) has a difference of squares. The elliptic equation (2) gives a nonzero lower bound for $\tau$. The hyperbolic equation (5) allows $\tau$ to approach zero because the matrix elements of a $\mathcal{P} \mathcal{T}$-symmetric Hamiltonian can be made large without violating the energy constraint $E_{+}-E_{-}=\omega$. The fact that $\tau$ can be made arbitrarily small may have applications in quantum computing.

We conclude with two comments. First, as $\alpha \rightarrow \frac{1}{2} \pi$ we get $\cos \alpha \rightarrow 0$. However, the energy constraint becomes $\omega^{2}=4 s^{2} \cos ^{2} \alpha$. Since $\omega$ is fixed, to have $\alpha$ approach $\frac{1}{2} \pi$, we must require $s \gg 1$. It follows from $\sin \alpha=(r / s) \sin \theta$ that $|r| \sim|s|$, so we must also require that $r \gg 1$. Thus, if $\tau \ll 1$, the matrix elements of the $\mathcal{P} \mathcal{T}$-symmetric Hamiltonian are large. Second, the result that $\tau=0$ does not violate the uncertainty principle. Both Hermitian and non-Hermitian $\mathcal{P} \mathcal{T}$-symmetric Hamiltonians share the properties that (i) the passage time is given by $\pi \hbar / \omega$, and (ii) $\Delta H \leq \omega / 2$.

To summarize, the key difference between the Hermitian and the non-Hermitian case is that $\left(\begin{array}{l}1 \\ 0\end{array}\right)$ and $\left(\begin{array}{l}0 \\ 1\end{array}\right)$ are orthogonal in the Hermitian case, but they have separation $\pi-2|\alpha|$ in the $\mathcal{P} \mathcal{T}$-symmetric case. This is because the Hilbert space metric of a $\mathcal{P} \mathcal{T}$ quantum theory depends on $H$. By choosing the parameter $\alpha$ properly, we create a wormhole-like effect in Hilbert space. That is, we find a path in Hilbert space from the initial state vector $\left|\psi_{I}\right\rangle$ to the final state vector $\left|\psi_{F}\right\rangle$ that is shorter than the Hermitian path. This is analogous to finding a wormhole in coordinate space. In short, what we have done here is to construct a "wormhole" in Hilbert space.

\section{Acknowledgements}

The author receives financial support from the U.S. Department of Energy.

\section{References}

[1] Bender C.M., Boettcher S., Meisinger P.N., PT -symmetric quantum mechanics, J. Math. Phys. 40 (1999), 2201-2209, quant-ph/9809072.

[2] Bender C.M., Boettcher S., Real spectra in non-Hermitian Hamiltonians having $\mathcal{P} \mathcal{T}$ symmetry, Phys. Rev. Lett. 80 (1998), 5243-5246, physics/9712001.

[3] Bender C.M., Introduction to $\mathcal{P} \mathcal{T}$-symmetric quantum theory, Contemp. Phys. 46 (2005), 277-292, quant-ph/0501052.

[4] Bender C.M., Making sense of non-Hermitian Hamiltonians, Rep. Prog. Phys. 70 (2007), 947-1018, hep-th/0703096.

[5] Dorey P., Dunning C., Tateo R., The ODE/IM correspondence, J. Phys. A: Math. Theor. 40 (2007), R205R283, hep-th/0703066. 
[6] Dorey P., Dunning C., Tateo R., Supersymmetry and the spontaneous breakdown of $\mathcal{P} \mathcal{T}$ symmetry, J. Phys. A: Math. Gen. 34 (2001), L391-L400, hep-th/0104119.

Dorey P., Dunning C., Tateo R., Spectral equivalences, Bethe ansatz equations, and reality properties in $\mathcal{P} \mathcal{T}$-symmetric quantum mechanics, J. Phys. A: Math. Gen. 34 (2001), 5679-5704, hep-th/0103051.

[7] Bender C.M., Brody D.C., Jones H.F., Complex extension of quantum mechanics, Phys. Rev. Lett. 89 (2002), 270401, 4 pages, quant-ph/0208076.

Bender C.M., Brody D.C., Jones H.F., Must a Hamiltonian be Hermitian?, Amer. J. Phys. 71 (2003), 1095-1102, hep-th/0303005.

[8] Bender C.M., Brody D.C., Jones H.F., Scalar quantum field theory with a complex cubic interaction, Phys. Rev. Lett. 93 (2004), 251601, 4 pages, hep-th/0402011.

[9] Bender C.M., Brandt S.F., Chen J.-H., Wang Q., The $\mathcal{C}$ operator in $\mathcal{P} \mathcal{T}$-symmetric quantum field theory transforms as a Lorentz scalar, Phys. Rev. D 71 (2005), 065010, 7 pages, hep-th/0412316.

[10] Wu T.T., Ground state of a Bose system of hard spheres, Phys. Rev. 115 (1959), 1390-1404.

[11] Hollowood T., Quantum solitons in affine Toda field theories, Nuclear Phys. B 384 (1992), 523-540, hep-th/9110010.

[12] Fisher M.E., Yang-Lee edge singularity and $\varphi^{3}$ field theory, Phys. Rev. Lett. 40 (1978), 1610-1613. Cardy J.L., Conformal invariance and the Yang-Lee edge singularity in two dimensions, Phys. Rev. Lett. 54 (1985), 1354-1356.

Cardy J.L., Mussardo G., S-matrix of the Yang-Lee edge singularity in two dimensions, Phys. Lett. B 225 (1989), 275-278.

Zamolodchikov A.B., Two-point correlation function in scaling Lee-Yang model, Nuclear Phys. B 348 (1991), 619-641.

[13] Brower R.C., Furman M.A., Moshe M., Critical exponents for the Reggeon quantum spin model, Phys. Lett. B 76 (1978), 213-219.

Harms B.C., Jones S.T., Tan C.-I., Complex energy spectra in reggeon quantum mechanics with quartic interactions, Nuclear Phys. 171 (1980), 392-412.

Harms B.C., Jones S.T., Tan C.-I., New structure in the energy spectrum of reggeon quantum mechanics with quartic couplings, Phys. Lett. B 91 (1980), 291-295.

[14] Günther U., Stefani F., Znojil M., MHD $\alpha^{2}$-dynamo, Squire equation and $\mathcal{P} \mathcal{T}$-symmetric interpolation between square well and harmonic oscillator, J. Math. Phys. 46 (2005), 063504, 22 pages, math-ph/0501069. Günther U., Samsonov B.F., Stefani F., A globally diagonalizable $\alpha^{2}$-dynamo operator, SUSY QM and the Dirac equation, J. Phys. A: Math. Theor. 40 (2007), F169-F176, math-ph/0611036.

[15] de Morisson Faria C.F., Fring A., Non-Hermitian Hamiltonians with real eigenvalues coupled to electric fields: from the time-independent to the time dependent quantum mechanical formulation, Laser Phys. 17 (2007), 424-437, quant-ph/0609096.

[16] Lee T.D., Some special examples in renormalizable field theory, Phys. Rev. 95 (1954), 1329-1334.

[17] Källén G., Pauli W., On the mathematical structure of T.D. Lee's model of a renormalizable field theory, Mat.-Fys. Medd. 30 (1955), no. 7.

[18] Bender C.M., Brandt S.F., Chen J.-H., Wang Q., Ghost busting: $\mathcal{P} \mathcal{T}$-symmetric interpretation of the Lee model, Phys. Rev. D 71 (2005), 025014, 11 pages, hep-th/0411064.

[19] Barton G., Introduction to advanced field theory, John Wiley \& Sons, New York, 1963, Chap. 12.

[20] Bender C.M., Mannheim P.D., No-ghost theorem for the fourth-order derivative Pais-Uhlenbeck oscillator model, Phys. Rev. Lett., to appear, arXiv:0706.0207.

[21] Mostafazadeh A., Exact $\mathcal{P} \mathcal{T}$-symmetry is equivalent to Hermiticity, J. Phys. A: Math. Gen. 36 (2003), 7081-7091, quant-ph/0304080.

[22] Buslaev V., Grecchi V., Equivalence of unstable anharmonic oscillators and double wells, J. Phys. A: Math. Gen. 26 (1993), 5541-5549.

Jones H.F., Mateo J., An equivalent Hermitian Hamiltonian for the non-Hermitian $-x^{4}$ potential, Phys. Rev. D 73 (2006), 085002, 4 pages, quant-ph/0601188.

Bender C.M., Brody D.C., Chen J.-H., Jones H.F., Milton K.A., Ogilvie M.C., Equivalence of a complex $\mathcal{P} \mathcal{T}$-symmetric quartic Hamiltonian and a Hermitian quartic Hamiltonian with an anomaly, Phys. Rev. D 74 (2006), 025016, 10 pages, hep-th/0605066.

[23] Carlini A., Hosoya A., Koike T., Okudaira Y., Quantum brachistochrone, Phys. Rev. Lett. 96 (2006), 060503, 4 pages, quant-ph/0511039. 
[24] Brody D.C., Hook D.W., On optimum Hamiltonians for state transformations, J. Phys. A: Math. Gen. 39 (2006), L167-L170, quant-ph/0601109.

[25] Bender C.M., Brody D.C., Jones H.F., Meister B.K., Faster than Hermitian quantum mechanics, Phys. Rev. Lett. 98 (2007), 040403, 4 pages, quant-ph/0609032.

[26] Assis P.E.G., Fring A., The quantum brachistochrone problem for non-Hermitian Hamiltonians, quant-ph/0703254.

Günther U., Rotter I., Samsonov B.F., Projective Hilbert space structures at exceptional points, J. Phys. A: Math. Theor. 40 (2007), 8815-8833, arXiv:0704.1291.

Martin D., Is $\mathcal{P} \mathcal{T}$-symmetric quantum mechanics just quantum mechanics in a non-orthogonal basis?, quant-ph/0701223.

Mostafazadeh A., Quantum brachistochrone problem and the geometry of the state space in pseudoHermitian quantum mechanics, Phys. Rev. Lett. 99 (2007), 130502, 4 pages, arXiv:0706.3844.

Günther U., Samsonov B.F., Non-unitary operator equivalence classes, the $\mathcal{P} \mathcal{T}$-symmetric brachistochrone problem and Lorentz boosts, arXiv:0709.0483.

Rotter I., The brachistochrone problem in open quantum systems, arXiv:0708.3891.

Mostafazadeh A., Physical meaning of Hermiticity and shortcomings of the composite (Hermitian + nonHermitian) quantum theory of Günther and Samsonov, arXiv:0709.1756. 